\title{
Evolución de las publicaciones en neurología clínica en España, Francia, Italia y Alemania en el período 2000-2009
}

\author{
Jesús Iñigo, Jorge Iriarte
}

Objetivo. Analizar la productividad y la visibilidad de las publicaciones españolas en la categoría temática de neurología clínica en el período 2000-2009 y compararlas con las correspondientes a Italia, Francia y Alemania.

Materiales y métodos. Se utilizó la base de datos Web of Science. El análisis (anual y quinquenal) se restringió a los documentos citables (artículos originales, revisiones y proceedings papers). Los indicadores bibliométricos utilizados fueron el número de publicaciones, las citas recibidas por las publicaciones y el índice $h$ de Hirsch. Se valoró también la pendiente anual de crecimiento $(b)$, el número de publicaciones por idioma y la colaboración internacional.

Resultados. En el período 2000-2009 hubo 46.114 publicaciones en neurología clínica, de las que 6.998 fueron publicaciones españolas $(h=75), 11.629$ italianas $(h=101), 9.745$ francesas $(h=102)$ y 20.143 alemanas $(h=124)$. El ritmo de incremento en el número total de publicaciones de España $(b=15)$ ha sido inferior al observado en Italia $(b=65)$, Alemania $(b=61)$ o Francia $(b=34)$. En el caso de las publicaciones en inglés, el ritmo de crecimiento fue superior para España $(b=37)$ que para Francia $(b=36)$, pero inferior al de Alemania $(b=54)$ e Italia $(b=65)$.

Conclusiones. Aunque el número total de publicaciones y el incremento observado son inferiores en España respecto a Italia, Francia o Alemania, las publicaciones españolas en neurología clínica muestran buenos indicadores de tendencia en lo referido a publicaciones en inglés y en colaboración internacional. Esta mejoría se asocia a una mayor visibilidad, que se pone de manifiesto al analizar por quinquenios las citas recibidas por las publicaciones españolas.

Palabras clave. Análisis bibliométrico. Comparación de países. Publicaciones en neurología.

\section{Introducción}

La actividad investigadora de un autor, grupo o conjunto de grupos debe y suele reflejarse en el número y calidad de sus publicaciones. Esta producción científica puede ser medida de forma bastante fidedigna analizando el número de publicaciones (productividad) y la frecuencia con que dichos trabajos son citados por otros (visibilidad e impacto) [1,2]. En los últimos años, se han producido avances tanto en el diseño de nuevos indicadores bibliométricos como en el desarrollo de bases de datos que permiten el análisis y la evaluación de esas publicaciones. Así, el número total de publicaciones científicas y el número total de citas recibidas por esas publicaciones son indicadores bibliométricos cada vez más utilizados de forma conjunta para valorar la cantidad y la calidad de la investigación que se realiza en un país [3].

Respecto al diseño de indicadores bibliométricos, los métodos clásicos para valorar una publicación incluían el factor de impacto de la revista [4] y el puesto de esa revista dentro del grupo de revistas del mismo tema en cuartiles (una revista del primer cuartil será aquélla que se encuentra en el 25\% de más impacto en la misma categoría temática). Otros métodos se basan en el número de citas recibidas por las publicaciones, y uno de los indicadores de este tipo más utilizados es el índice $h$ (de Hirsch), que consiste en ordenar las publicaciones del autor por el número de citas recibidas, y tomar el número del artículo que recibe al menos el mismo número en citas [5].

Ninguno de los indicadores bibliométricos descritos está libre de críticas [6,7]. Las limitaciones del factor de impacto derivan de que es un indicador apropiado para valorar la visibilidad y repercusión de la revista en general, pero no de un artículo en concreto. Las limitaciones del índice $h$ proceden del hecho de depender de los años de las publicaciones, premiando a los autores con experiencia, pues los artículos van recibiendo las citas con el paso de los años; además, es poco selectivo, pues puntúa igual al primer autor que a los demás, ya
Consejería de Sanidad; Comunidad de Madrid; Madrid (J. Iñigo) Clínica Universidad de Navarra; Universidad de Navarra; Pamplona, Navarra, España (J. Iriarte).

Correspondencia: Dr. Jorge Iriarte Franco Clínica Universidad de Navarra. Universidad de Navarra. Pío XII, 36 E-31080 Pamplona (Navarra).

Fax: +34948172294.

E-mail: jiriarte@unav.es

Presentado en forma de póster en la LXIII Reunión Anual de la Sociedad Española de Neurología (Barcelona, 15-19 de noviembre de 2011).

Aceptado tras revisión externa: 23.09.11.

Cómo citar este artículo: Iñigo J, Iriarte J. Evolución de las publicaciones en neurología clínica en España, Francia, Italia y Alemania en el período 2000-2009. Rev Neurol 2011; 53: 591-9.

(c) 2011 Revista de Neurología 
que el artículo se asigna a cada uno de los autores del mismo modo. Otro problema es que no es un sistema lineal, sino que resulta fácil aumentar el valor del indicador al principio, pero a partir de un momento es muy difícil añadir publicaciones de relevancia (y, por ello, es menos discriminante en el rango medio de valores). Asimismo, existen áreas médicas más citadas que otras [1]. Sin embargo, y a pesar de estas limitaciones, el índice $h$ es cada más usado para evaluar la trayectoria de un profesional o de grupos de investigadores, de instituciones y de países [8-10].

Respecto a las bases de datos, hasta hace relativamente poco tiempo el uso de la Web of Science era el único medio disponible para realizar estudios de citaciones. En España, la Fundación Española de Ciencia y Tecnología ha patrocinado y promovido el acceso abierto a la Web of Knowledge de Thomson-ISI, de la que forma parte la Web of Science, y ésta es la base de datos más usada para valorar el número de citas recibidas por las publicaciones (http://www.accesowok.fecyt.es/). Desde el año 2004 existen otras bases de datos que permiten realizar este tipo de estudios [11], incluyendo Scopus (suscripción de pago) y Google Scholar (gratuita), y los resultados de las búsquedas no son idénticos [12].

Dado que las actuales bases de datos bibliográficas permiten obtener información no sólo por autor, sino también por centros o incluso países, para una o más ramas de la medicina [13], nos hemos planteado este trabajo sobre las publicaciones en neurología clínica. Nos hemos propuesto analizar la productividad y la visibilidad de las publicaciones españolas en la categoría temática de neurología clínica en los últimos diez años y compararlas con las correspondientes a Italia, Francia y Alemania, países vecinos y de un entorno sanitario, cultural y técnico del nivel del español [3].

\section{Materiales y métodos}

\section{Realización de la búsqueda de las publicaciones}

La búsqueda bibliográfica se ha realizado en la base de datos Science Citation Index Expanded perteneciente a la Web of Science del Institute for Scientific Information (ISI Web of Knowledge) y se llevó a cabo en abril de 2010. Se obtuvieron las publicaciones de la categoría temática de neurología clínica (Clinical Neurology) de España, Italia, Francia y Alemania entre los años 2000 y 2009, ambos incluidos.
En el caso de estudios con participación de dos o más de estos países, los documentos se adscribieron a cada uno de ellos. Los datos se obtuvieron por país y año.

\section{Datos de las publicaciones evaluados}

En la búsqueda se han incluido artículos originales, artículos de revisión y trabajos presentados en congresos (proceedings papers). En la presentación de datos se han considerado los valores globales y los datos según el tipo de artículo. Asimismo, se ha realizado el análisis distinguiendo el número de artículos publicados en lengua inglesa, suponiendo que estos artículos denotan una mayor difusión, y probablemente mayor número de citas recibidas. También se ha analizado el número de artículos fruto de colaboración internacional como dato de interés para valorar la capacidad de los grupos para las relaciones científicas con grupos de otros países.

\section{Factor de impacto, índice $h$ y número de citas}

A partir de la base de datos Journals of Citations Reports (JCR) de Thomson-ISI se obtuvieron los factores de impacto de las revistas de la categoría temática de neurología clínica para cada uno de los años del estudio y se clasificaron las revistas en cuartiles en función de dicho factor. Se calculó el número total de citas recibidas por las publicaciones de cada país y año. El índice $h$ acumulado de un período (por ejemplo, 2000-2002) se calculó considerando todas las publicaciones de estos años y las citas recibidas por esas publicaciones hasta la fecha de la búsqueda bibliográfica.

\section{Estudio estadístico}

La valoración de los datos de la evolución del número de artículos y de su visibilidad ha sido únicamente descriptiva. Para cada uno de los indicadores (con excepción del índice $h$, que no sigue una distribución lineal) y para cada país, se ajustaron los datos anuales a una recta, obteniéndose el valor de su pendiente $(b)$ como parámetro para cuantificar el ritmo de crecimiento de los indicadores. Además, se han comparado dos quinquenios (20002004 y 2005-2009), para ver la evolución interna. Dada la naturaleza de los datos (frecuencias e índices correspondientes a los últimos diez años) y los objetivos del trabajo, se ha considerado suficiente presentar los datos de forma descriptiva con tablas y gráficos. 


\section{Resultados}

\section{Número de publicaciones}

En el período comprendido entre los años 2000 y 2009, hubo 46.114 publicaciones españolas, italianas, francesas o alemanas en la categoría temática de neurología clínica, de las que 45.197 (98\%) correspondieron a 192 revistas científicas diferentes y las 917 restantes a otras fuentes (principalmente libros de congresos científicos, que incluyen proceedings papers). De las 46.114 publicaciones totales, $6.998(15,2 \%)$ fueron publicaciones españolas, 11.629 $(25,7 \%)$ italianas, $9.745(21,1 \%)$ francesas y 20.143 $(43,7 \%)$ alemanas.

La pendiente de crecimiento en el número de publicaciones fue más alta en el caso de Italia que en los de Alemania, Francia y España $(b=65,61,34$ y 15 , respectivamente). La distribución anual de las publicaciones de España, Italia, Francia y Alemania se muestra en la figura 1.

\section{Tipo de publicación}

De las 6.998 publicaciones españolas en neurología clínica, $5.636(80,5 \%)$ correspondieron a artículos originales, $849(12,1 \%)$ a revisiones y $513(7,3 \%)$ a proceedings papers. Entre las 11.629 publicaciones italianas, $9.493(81,6 \%)$ fueron artículos originales, mientras que $903(7,8 \%)$ fueron revisiones y 1.233 $(10,6 \%)$ proceedings papers. La distribución de las 9.745 publicaciones francesas fue muy parecida: $7.832(80,4 \%)$ artículos originales, 805 (8,3\%) revisiones y $1.108(11,4 \%)$ proceedings papers. En cambio, entre las 20.143 publicaciones alemanas hubo menos proceedings papers -1.305 (6,5\%)- y ligeramente más artículos originales $-16.850(83,7 \%)-$ y revisiones $-1.988(9,9 \%)$-.

La distribución anual de los artículos originales españoles, italianos, franceses y alemanes en la categoría temática de neurología clínica se muestra en la figura 2. En los artículos originales, en el período 2000-2009, el ritmo de incremento anual fue superior para Italia $(b=58)$ y Alemania $(b=56)$, que para Francia $(b=37)$ y España $(b=15)$. En el caso de las revisiones, el aumento anual fue mayor para Alemania que para Italia, Francia y España ( $b=16,10,8$ y 6 , respectivamente).

\section{Publicaciones en inglés}

La distribución anual de las publicaciones españolas, italianas, francesas y alemanas en neurología clínica y redactadas en inglés se muestra en la figura 3.
Figura 1. Número anual de publicaciones de España, Italia, Francia y Alemania en neurología clínica. Período 2000-2009.

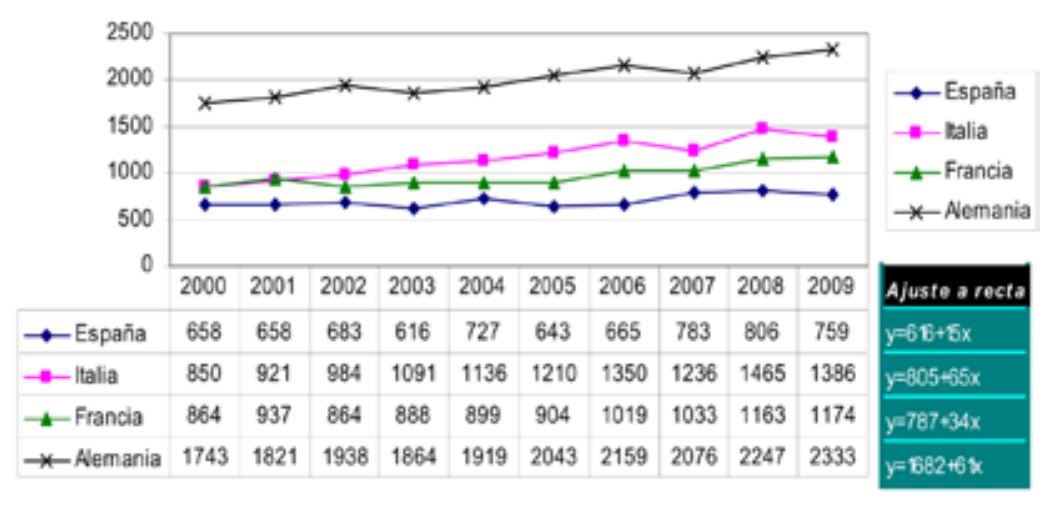

Figura 2. Número anual de artículos originales de España, Italia, Francia y Alemania en neurología clínica. Período 2000-2009.

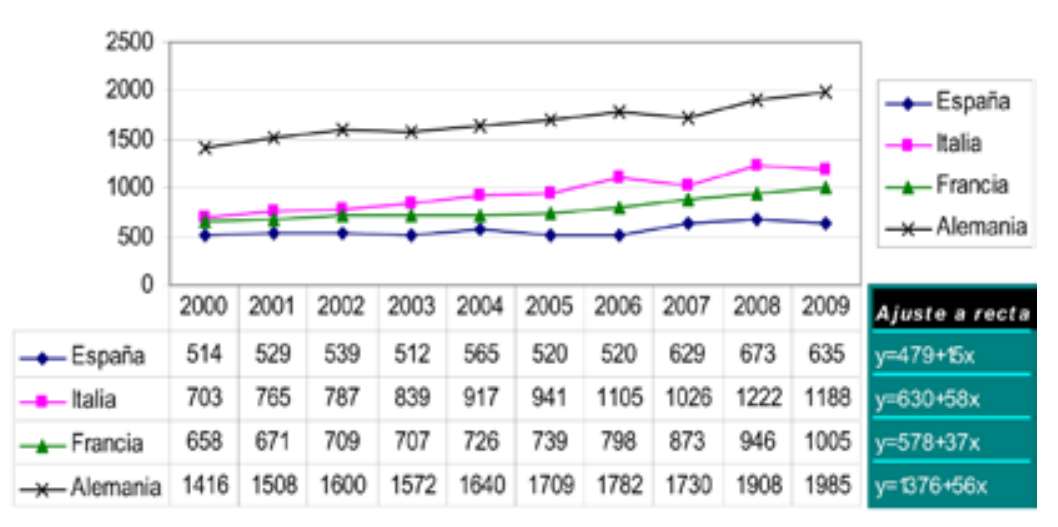

Figura 3. Número anual de publicaciones españolas, italianas, francesas y alemanas en neurología clínica redactadas en inglés. Período 2000-2009.

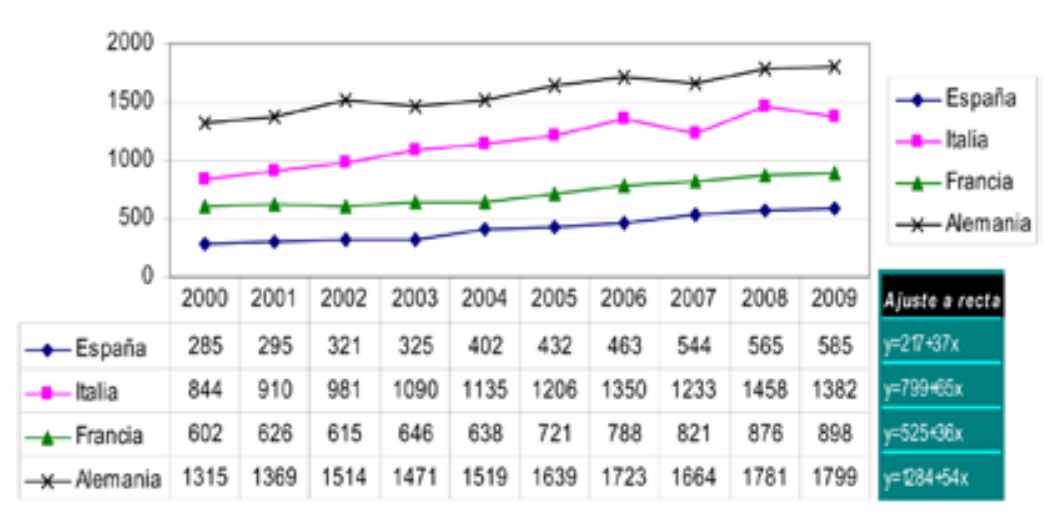


Figura 4. Número anual de publicaciones españolas, italianas, francesas y alemanas en neurología clínica en colaboración internacional. Período 2000-2009.

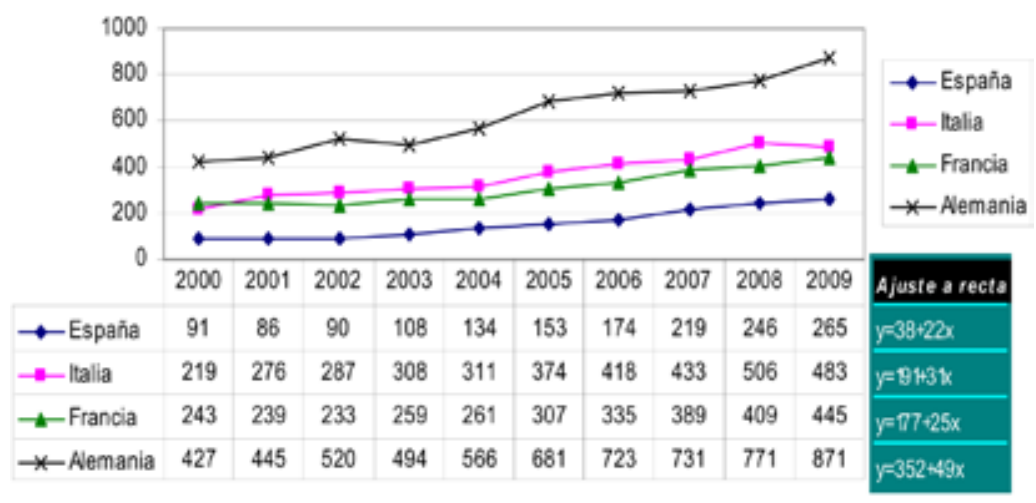

Figura 5. Citas acumuladas recibidas por las publicaciones españolas, italianas, francesas y alemanas en neurología clínica. Período 2000-2009.

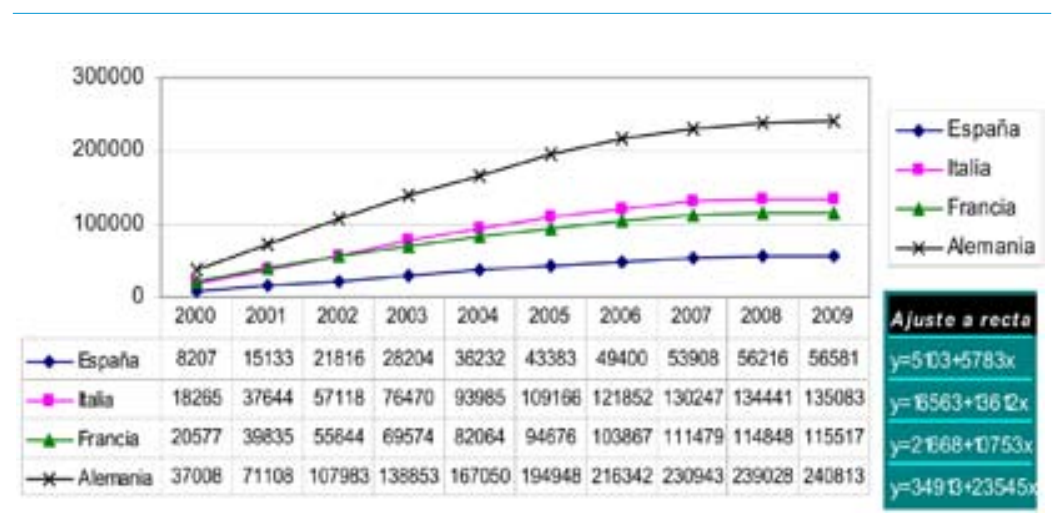

Figura 6. Índice $h$ acumulado de las publicaciones españolas, italianas, francesas y alemanas en neurología clínica. Período 2000-2009.

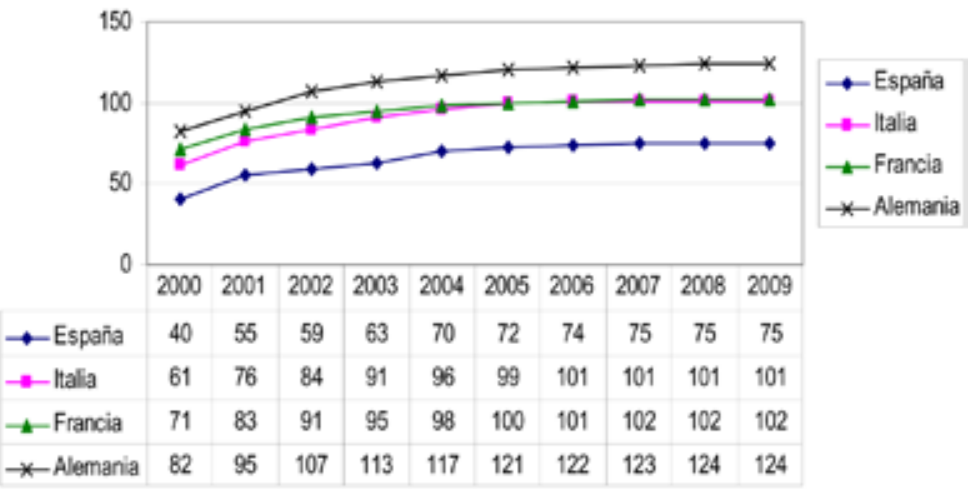

De las 6.998 publicaciones españolas en neurología clínica, $4.217(60,3 \%)$ estaban redactadas en inglés, $2.706(38,7 \%)$ en español y $75(1,1 \%)$ en otros idiomas. La proporción era más alta en las publicaciones italianas, ya que, de las 11.629 publicaciones, $11.589(99,7 \%)$ eran en inglés. Las cifras de las publicaciones de origen francés y alemán en inglés son intermedias entre estas cifras: $7.231(74,2 \%)$ y 15.794 (78,4\%), respectivamente. La pendiente de crecimiento en el número de publicaciones en inglés fue más alta en el caso de Italia $(b=65)$ que en los de Alemania $(b=54)$, España $(b=37)$ y Francia $(b=36)$.

\section{Publicaciones realizadas en colaboración internacional}

Entre las 6.998 publicaciones españolas en neurología clínica, $1.566(22,4 \%)$ se realizaron en colaboración internacional. De la misma forma, se realizaron en colaboración internacional $3.615(31,1 \%)$ de las 11.629 publicaciones italianas, $3.120(32 \%)$ de las 9.745 francesas y $6.229(30,9 \%)$ de las 20.143 alemanas. La distribución anual de las publicaciones españolas, italianas, francesas y alemanas en neurología clínica, realizadas en colaboración internacional, se muestra en la figura 4. El ritmo de incremento anual de las publicaciones alemanas en colaboración internacional fue superior al de las publicaciones italianas, francesas y españolas, con valores $b$ de $49,31,25$ y 22 , respectivamente.

\section{Indicadores bibliométricos}

La evolución del número de citas recibidas por las publicaciones españolas, italianas, francesas y alemanas en neurología clínica se muestra en la figura 5. En el período 2000-2009, el ritmo de crecimiento anual de este indicador ha sido mayor para Alemania que para Italia, Francia y España $(b=23.545$, $13.612,10.753$ y 5.783 , respectivamente).

En la figura 6 se muestra la distribución del índice $h$ acumulado en el período 2000-2009 para el conjunto de publicaciones de los cuatro países en neurología clínica. Alemania consiguió un índice $h$ acumulado más alto $(h=124)$, Italia y Francia presentaron un valor similar $(h=101$ y 102 , respectivamente), y en el caso de España fue claramente inferior $(h=75)$. Estas diferencias se observan también para los artículos originales (aunque con Francia superando a Italia) y en las revisiones (en este caso, con Italia superando a Francia) por separado (Tabla I). En el caso de los proceedings papers, las diferencias en los valores del índice $h$ son menores.

El índice $h$ acumulado de las publicaciones en inglés fue el mismo que el de las publicaciones tota- 
Tabla I. Número de publicaciones e índice $h$ acumulado de las publicaciones españolas, italianas, francesas y alemanas, según tipo de publicación, idioma y colaboración internacional. Período 2000-2009.

\begin{tabular}{|c|c|c|c|c|c|c|c|c|c|}
\hline & & \multicolumn{2}{|c|}{ España } & \multicolumn{2}{|c|}{ Italia } & \multicolumn{2}{|c|}{ Francia } & \multicolumn{2}{|c|}{ Alemania } \\
\hline & & $n$ & $\begin{array}{c}h \\
\text { acumulado }\end{array}$ & $n$ & $\begin{array}{c}h \\
\text { acumulado }\end{array}$ & $n$ & $\begin{array}{c}h \\
\text { acumulado }\end{array}$ & $n$ & $\begin{array}{c}h \\
\text { acumulado }\end{array}$ \\
\hline \multirow{3}{*}{$\begin{array}{l}\text { Tipo de } \\
\text { publicación ISI }\end{array}$} & Artículo & 5.636 & 71 & 9.493 & 90 & 7.832 & 97 & 16.850 & 115 \\
\hline & Proceedings paper & 513 & 34 & 1.233 & 39 & 1.108 & 40 & 1.305 & 44 \\
\hline & Revisión & 849 & 34 & 903 & 57 & 805 & 53 & 1.988 & 72 \\
\hline \multirow{2}{*}{$\begin{array}{l}\text { Idioma de } \\
\text { la publicación }\end{array}$} & Inglés & 4.217 & 75 & 11.589 & 101 & 7.231 & 102 & 15.794 & 124 \\
\hline & Otros $^{a}$ & 2.781 & 14 & 40 & 4 & 2.514 & 15 & 4.349 & 22 \\
\hline \multirow{2}{*}{$\begin{array}{l}\text { Colaboración } \\
\text { internacional }\end{array}$} & Sí & 1.555 & 64 & 3.511 & 92 & 3.120 & 91 & 6.229 & 109 \\
\hline & No & 5.443 & 52 & 8.118 & 69 & 6.625 & 75 & 13.914 & 95 \\
\hline Total & & 6.998 & 75 & 11.629 & 101 & 9.745 & 102 & 20.143 & 124 \\
\hline
\end{tabular}

a España cuenta con 2.706 publicaciones en español, Francia con 2.488 publicaciones en francés y Alemania con 4.309 en alemán.

les para los cuatro países. Las publicaciones realizadas en idiomas diferentes al inglés presentaron índices $h$ acumulados muy inferiores. Las publicaciones de cada uno de los cuatro países realizadas en colaboración internacional presentaron un índice $h$ acumulado superior al de las realizadas sin colaboración internacional, con diferencias más importantes para Italia y Francia (de 23 y 21 puntos, respectivamente) que para España y Alemania (12 y 14 puntos, respectivamente). Para las publicaciones realizadas sin colaboración internacional, Francia supera a Italia en el índice $h$ acumulado, a pesar de que su número de publicaciones es inferior.

\section{Comparación del quinquenio 2000-2004 con el quinquenio 2005-2009}

Los datos de la evolución de los datos bibliométricos por países en el primer y segundo quinquenio se recogen en la tabla II. Se muestra el número total de publicaciones y de citas, el índice $h$ del período, el número y porcentaje de artículos por cuartiles, el número de artículos citados 20 o más veces (los del primer quinquenio) o más de siete veces (los del segundo quinquenio), los artículos no citados y los artículos en colaboración con centros de otros países. Entre las 5.037 publicaciones citadas 20 veces o más en el quinquenio $2000-2004$, un $69,7 \%$ se publicó en revistas del primer cuartil, un $19,9 \%$ en el segundo, un $8,1 \%$ en el tercero y un 2,3\% en el último cuartil. Expresado de forma inversa, un 49,6\% de las publicaciones en revistas del primer cuartil en ese mismo quinquenio fueron citadas 20 veces o más, frente a un $26,9 \%$, un $11,3 \%$ y un $2,2 \%$ de las publicaciones en revistas del segundo, tercero y último cuartil.

De los datos de la tabla II destaca la mejoría de España en relación con los otros países en el número de artículos en el primer cuartil. Aunque seguimos por debajo del resto, ha habido una mejoría del 9,5\%, mientras que Francia ha mejorado el 1,6\%, y Alemania e Italia han bajado. Además, en el porcentaje de artículos en colaboración internacional, también se ha crecido, hasta casi igualar al resto. En el porcentaje de artículos no citados, España está prácticamente al nivel de los otros países, cuando en el quinquenio anterior estaba claramente por encima.

\section{Discusión}

Alemania, Francia, Italia y España se encuentran entre los diez países del mundo con mayor número de artículos científicos publicados en el quinquenio 2004-2008, considerando todas las áreas de la ciencia. Estos cuatro países se encuentran también entre los diez países con mayor número de citas reci- 
Tabla II. Publicaciones en neurología clínica de España, Italia, Francia y Alemania en los períodos 2000-2004 y 2005-2009 a.

\begin{tabular}{|c|c|c|c|c|c|}
\hline & Cuatro países & España & Italia & Francia & Alemania \\
\hline \multicolumn{6}{|l|}{ Período 2000-2004 } \\
\hline Total de publicaciones & 19.627 & 3.108 & 4.528 & 4.142 & 8.633 \\
\hline Índice $h$ del período & 139 & 70 & 96 & 98 & 116 \\
\hline En primer cuartil & $7.078(36,1 \%)$ & $738(23,7 \%)$ & $1.988(43,9 \%)$ & $1.517(36,6 \%)$ & $3.353(38,8 \%)$ \\
\hline En primer y segundo cuartil & $10.804(55,0 \%)$ & $1.159(37,3 \%)$ & $3.121(68,9 \%)$ & $2.168(52,3 \%)$ & $5.019(58,1 \%)$ \\
\hline En último cuartil & $5.209(26,5 \%)$ & $1.690(54,4 \%)$ & $359(7,9 \%)$ & $1.304(31,5 \%)$ & $1.894(21,9 \%)$ \\
\hline Citadas $\geq 20$ veces $^{b}$ & $5.037(25,7 \%)$ & $480(15,4 \%)$ & $1.397(30,9 \%)$ & $1.137(27,5 \%)$ & $2.471(28,6 \%)$ \\
\hline No citadas & $2.109(10,7 \%)$ & $503(16,2 \%)$ & $191(4,2 \%)$ & $534(12,9 \%)$ & $894(10,4 \%)$ \\
\hline En colaboración internacional & $4.487(22,9 \%)$ & $476(15,3 \%)$ & $1.326(29,3 \%)$ & $1.137(27,5 \%)$ & $2.332(27,0 \%)$ \\
\hline Total de citas recibidas & 345.456 & 36.232 & 93.985 & 82.064 & 167.050 \\
\hline \multicolumn{6}{|l|}{ Período 2005-2009 } \\
\hline Total de publicaciones & 23.985 & 3.592 & 6.374 & 5.070 & 10.488 \\
\hline Índice $h$ del período & 82 & 47 & 57 & 55 & 73 \\
\hline En primer cuartil & $8.275(34,5 \%)$ & $1.156(32,2 \%)$ & $2.453(38,5 \%)$ & $1.939(38,2 \%)$ & $3.699(35,3 \%)$ \\
\hline En primer y segundo cuartil & $14.612(60,9 \%)$ & $1.999(55,7 \%)$ & $4.367(68,5 \%)$ & $3.071(60,6 \%)$ & $6.517(62,1 \%)$ \\
\hline En último cuartil & $5.791(24,1 \%)$ & $1.083(30,2 \%)$ & $770(12,1 \%)$ & $1.378(27,2 \%)$ & $2.632(25,1 \%)$ \\
\hline Citadas $\geq 7$ veces $^{c}$ & $6.147(25,6 \%)$ & $793(22,1 \%)$ & $1.780(27,9 \%)$ & $1.324(26,1 \%)$ & $3.034(28,9 \%)$ \\
\hline No citadas & $7.260(30,3 \%)$ & $1.133(31,5 \%)$ & $1.727(27,1 \%)$ & $1.679(33,1 \%)$ & $2.956(28,2 \%)$ \\
\hline En colaboración internacional & $7.220(30,1 \%)$ & $1.043(29,0 \%)$ & 2.169 (34\%) & $1.842(36,3 \%)$ & $3.705(35,3 \%)$ \\
\hline Total de citas recibidas & 14.5247 & 20.349 & 41.098 & 33.453 & 73.763 \\
\hline
\end{tabular}

a Análisis realizado con $43.612(94,6 \%)$ de las 46.114 publicaciones totales. Las 2.502 publicaciones restantes no se incluyen porque no se realizaron en revistas con factor de impacto asignado, sino en otras fuentes (libros de congresos, series o revistas sin factor de impacto); ${ }^{b}$ Las publicaciones que fueron citadas 20 veces o más se encuentran en el cuartil superior de la distribución del número de citas recibidas; ' ${ }^{c}$ Las publicaciones que han sido citadas siete veces o más están en el cuartil superior de citas recibidas.

bidas (respecto a este indicador, China y España han desplazado de los diez primeros puestos a Suiza y Australia, que se situaban por delante en el quinquenio 1999-2003) [14]. Si los distintos países del mundo se colocan en una gráfica en la que se representa en un eje el producto interior bruto y en el otro las citas recibidas por sus publicaciones, Alemania, Francia, Italia y España aparecen muy cercanos en la distribución resultante [3], y, por tanto, son países que pueden considerarse comparables desde un punto de vista bibliométrico.

El presente trabajo es una revisión retrospectiva vía Web of Science de la evolución de las publicaciones en neurología clínica en los últimos 10 años en España y otros tres países europeos (Francia, Italia y
Alemania). Una comparación más exacta de los indicadores bibliométricos obtenidos puede realizarse utilizando factores de corrección por población (multiplicando por 1,2 los valores de Francia, por 1,4 los de Italia y por 1,8 los de España) o por producto interior bruto (multiplicando por 1,3 los valores de Francia, por 1,6 los de Italia y por 2,3 los de España).

El índice $h$, utilizado en el presente estudio, fue diseñado por Hirsch para evaluar las publicaciones de un único investigador [5], pero muy pronto se extendió su uso a ámbitos más amplios y se propusieron gran cantidad de variantes derivadas de su cálculo. Una de las variantes se ha centrado en la posibilidad de utilizarlo como base para la obtención de una serie sucesiva de índices $h$ que permi- 
tiera, a partir de las publicaciones de los investigadores de una misma institución, obtener índices $h$ asociados a esa institución y determinar posteriormente el índice $h$ de un país [8]. Otros investigadores han abordado el uso del índice $h$ y de sus derivados para evaluar las publicaciones de distintas universidades en las mismas áreas y categorías temáticas. Así, se han publicado los valores del índice $h$ de las universidades europeas en el área de biomedicina y para las distintas categorías temáticas (por ejemplo, la Universidad de Oxford presentó en el quinquenio 2002-2006 un índice $h$ de 105 en biomedicina, con valores del índice de 60 en medicina general e interna, de 31 en enfermedades infecciosas y de 29 en cardiología) [15]. Otros autores han publicado los resultados del cálculo del índice $h$ para diversos países en distintas áreas y categorías temáticas $[9,10]$. La base de datos Scopus permite, mediante la página web de Scimago (www.scimagojr.com), el cálculo del índice $h$ para cualquier área y categoría temática, facilitando, además, la comparación entre los distintos países. Así, en el área temática de medicina, España ocupa el puesto 13 según el índice $h$ de las publicaciones del período 1996-2008 [16]. En el período 1996-2009, el índice $h$ acumulado en la categoría temática de neurología clínica es de 138 para Alemania, de 116 para Francia, de 109 para Italia y de 82 para España (valores ligeramente superiores a los obtenidos en nuestro estudio, basado en Web of Science y con un menor período de análisis).

Aunque es conocido que la búsqueda por la Web of Science no es exacta, y que estos resultados pueden tener alguna limitación por primar las revistas anglosajonas [6,17], esta herramienta es, junto con Scopus, la utilizada más habitualmente en los estudios bibliométricos en los que se considera el número de citas $[2,13,14,18-20]$. Esta limitación, en todo caso, afectaría de la misma forma a los cuatro países que se comparan. Respecto a los indicadores bibliométricos elegidos, en nuestro estudio hemos optado por el número de publicaciones, el número de citas recibidas y el índice $h$. Otros autores han optado por estudiar la vida media de citación, el índice de inmediatez o el número de artículos en el top 10 o en el top $3[18,19,21]$. En un estudio sobre publicaciones en obesidad en España, los autores se centraron en el número total de publicaciones y las publicaciones en inglés [22]. Otros estudios, en cambio, seleccionan otros criterios y se centran en el factor de impacto total y el número de artículos con factor de impacto superior a 3 [23].

Los resultados de nuestro estudio indican, en valores absolutos, una mayor capacidad de publica- ción y un mayor número de citas recibidas en Alemania, seguida de Italia, a continuación Francia y finalmente España. Llama la atención que Italia, teniendo menos habitantes que Francia, esté por encima en número de publicaciones, en publicaciones en inglés y también en citas recibidas, y además, su ritmo de crecimiento es el más alto de los cuatro países considerados. La tendencia global en el número de publicaciones es en los cuatro países hacia el aumento, tanto en originales como en revisiones.

$\mathrm{Al}$ examinar las publicaciones en inglés, se aprecia que el ritmo de crecimiento en España es superior al de Francia, a pesar de que las publicaciones totales francesas crecen a un ritmo dos veces mayor que las españolas. Respecto a la colaboración internacional, España es el único de los cuatro países en el que el ritmo de crecimiento de estas publicaciones supera al de crecimiento global. En el segundo quinquenio, el porcentaje de publicaciones españolas realizadas en colaboración internacional se ha duplicado respecto al primero, mientras que el incremento observado en los otros tres países ha sido menor. Estos datos reflejan el resultado positivo del esfuerzo realizado en España en los últimos años para mejorar la calidad de las publicaciones, aunque sería deseable que las publicaciones realizadas en español también tuvieran una mayor visibilidad.

Analizando el índice $h$, se ve que hubo un crecimiento hasta 2005 y que, después, el crecimiento ha sido muy lento en los cuatro países. Esto se debe a lo difícil que es aumentar el índice $h$ cuando su número es alto, pues hacen falta publicaciones con muchas citas para aumentar este valor, por lo cual deben ser muy buenas y debe pasar cierto tiempo hasta conseguir las citas requeridas para aumentar el índice $h$ en un punto [1]. Llama la atención que España se queda estancada en valores inferiores al resto, y que Francia e Italia se igualan. Esto implica que las publicaciones francesas que reciben más citas son un número mayor que las italianas, y que entre las publicaciones italianas hay algunas que concentran la mayoría de las citas y, por ello, el índice $h$ no crece tanto como correspondería a las citas recibidas globalmente. Con respecto a España, es preocupante que no haya publicaciones de más calidad para incrementar este índice $h$ 'nacional.'

La mejoría en las publicaciones en neurología clínica descrita en este trabajo es coherente con los resultados publicados sobre la evolución de las publicaciones españolas en biomedicina y ciencias de la salud $[13,20]$. La mejoría sigue de año en año, tanto en número de publicaciones como en calidad. Las revistas en castellano reciben menos citas, y en ellas tiene menor peso la colaboración internacio- 
nal [24], por lo que se está pasando a publicar más en las revistas extranjeras [25-27]. En cambio, las publicaciones en Italia son habitualmente en inglés o bilingües, y ello explica que sean más citadas.

El análisis bibliográfico realizado ha servido para mostrar la tendencia en el número de publicaciones en neurología clínica, y su visibilidad. Objetivar estos datos de las publicaciones y estudiar la cooperación internacional en las publicaciones puede servir para fijar una posición de la investigación y publicaciones en nuestro país con datos reales. Presentados estos datos de forma fría, se confirma que estamos por debajo de Alemania, Italia y Francia. Sin embargo, si se leen despacio, se aprecia que el avance realizado en este último decenio ha sido muy llamativo. Estos datos son incluso mejores si se compara la evolución del segundo quinquenio de la década en relación con el primero: las publicaciones de los neurólogos españoles han aumentado y han mejorado muy significativamente, así como la colaboración con centros de otros países. Si se corrigieran los datos por población, no estaríamos muy lejos de Francia. La información obtenida en este trabajo muestra unos resultados positivos para nuestro país, que deben seguir animando a los neurólogos españoles a seguir en la línea de estos últimos años para alcanzar el nivel de los países vecinos.

Bibliografía

1. Gisbert JP, Panés J. Publicación científica, indicadores bibliométricos e índice $h$ de Hirsch. Gastroenterol Hepatol 2009; 32: 140-9.

2. Dorta-Contreras AJ, Arencibia-Jorge R, Martí-Lahera Y, Araujo-Ruiz JA. Productividad y visibilidad de los neurocientíficos cubanos: estudio bibliométrico del período 2001 2005. Rev Neurol 2008; 47: 355-60.

3. King DA. The scientific impact of nations. Nature 2004; 430 : 311-6.

4. Garfield E. The history and meaning of the journal impact factor. JAMA 2006; 295: 90-3

5. Hirsch JE. An index to quantify an individual's scientific research output. Proc Natl Acad Sci U S A 2005; 102: 16569-72.

6. Camí J. Impactolatría: diagnóstico y tratamiento. Med Clin (Barc) 1997; 109: 515-24.

7. Camí J. Evaluación de la investigación biomédica. Med Clin (Barc) 2001; 117: 510-3

8. Schubert A. Successive h-indices. Scientometrics 2007; 70: 201-5.

9. Csajbók E, Berhidi A, Vasas L, Schubert A. Hirsch-index for countries based on Essential Science Indicators data. Scientometrics 2007; 73: 91-117.

10. Imperial J, Rodríguez-Navarro A. Usefulness of Hirsch's h-index to evaluate scientific research in Spain. Scientometrics 2007; 71: 271-82.

11. Roth DL. The emergence of competitors to the Science Citation Index and the Web of Science. Curr Sci 2005; 89: 1531-6.

12. Kulkarni AV, Aziz B, Shams I, Busse JW. Comparisons of citations in Web of Science, Scopus, and Google Scholar for articles published in general medical journals. JAMA 2009; 302: 1092-6.

13. Méndez-Vásquez RI, Suñén-Pinyol E, Cervelló R, Camí J. Mapa bibliométrico de España 1996-2004: biomedicina y ciencias de la salud. Med Clin (Barc) 2008; 130: 246-53.

14. The Royal Society. Knowledge, networks and nations: global scientific collaboration in the 21st century. London: The Royal Society-Elsevier; 2011.

15. Sypsa V, Hatzakis A. Assessing the impact of biomedical research in academic institutions of disparate size. BMC Med Res Methodol 2009; 9: 33.

16. Spiroski M. Country rank, journal rank and H-index in the field of Medicine in the Republic of Macedonia (1996-2008) using data from Scimago. Macedonian J Med Sci 2010; 3: 99-108.

17. Seglen PO. Citations and journal impact factors: questionable indicators of research quality. Allergy 1997; 52: 1050-6.

18. Durieux V, Gevenois PA. Bibliometric indicators: quality measurements of scientific publication. Radiology 2010; 255: 342-51.

19. Téllez H, Vadillo JM. Bibliometric study of journal publications on analytical chemistry 2000-2007: publication productivity and journal preferences by country. Anal Bioanal Chem 2010; 397: $1477-84$.

20. Camí J, Suñén-Piñol E, Méndez-Vásquez R. Mapa bibliométrico de España 1994-2002: biomedicina y ciencias de la salud. Med Clin (Barc) 2005; 124: 93-101.

21. Smith DR. A longitudinal analysis of bibliometric and impact factor trends among the core international journals of nursing, 1977-2008. Int J Nurs Stud 2010; 47: 1491-9.

22. Vioque J, Manuel RJ, Navarrete-Muñoz EM, García DLH. Producción científica española en obesidad a través de PubMed (1988-2007). Gac Sanit 2010; 24: 225-32.

23. Leonardelli $S$, Belmin J. International publications from the French geriatric teams: evolution in the course of last 22 years. J Nutr Health Aging 2008; 12: 285-8.

24. González-Alcaide G, Alonso-Arroyo A, González de Dios J, Sempere AP, Valderrama-Zurián JC, Aleixandre-Benavent R. Redes de coautoría y colaboración institucional en Revista de Neurología. Rev Neurol 2008; 46: 642-51.

25. González de Dios J, Sempere AP, Aleixandre-Benavent R. Las publicaciones biomédicas en España a debate (I): estado de las revistas neurológicas. Rev Neurol 2007; 44: 32-42.

26. González de Dios J, Sempere AP, Aleixandre-Benavent R. Las publicaciones biomédicas en España a debate (II): las 'revoluciones' pendientes y su aplicación a las revistas neurológicas. Rev Neurol 2007; 44: 101-12.

27. González de Dios J, Valderrama-Zurián JC, González-Alcaide G, Sempere AP, Bolaños-Pizarro M, Aleixandre-Benavent R. Aproximación al 'impacto' de las revistas biomédicas en ciencias neurológicas: estudio de los indicadores bibliométricos en el Journal Citation Reports-Science Citation Index 2006. Rev Neurol 2009; 48: 117-28.

Evolution of the literature on clinical neurology in Spain, France, Italy and Germany over the period 2000-2009

Aim. This study analyzes the productivity and visibility of Spanish publications in the area of clinical neurology in the period 2000-2009 and compared with those for Italy, France and Germany.

Materials and methods. We used the database Web of Science. The analysis (annual and in five-year) was restricted to the 
citable documents (original articles, reviews and proceedings papers). Bibliometric indicators used were the number of publications, citations received by publications and Hirsch's $h$-index. We also assessed the slope of the annual growth rate (b), the number of publications by language and the international collaboration.

Results. In the period 2000-2009 there were 46,114 publications in neurology clinic of which 6,998 were Spanish publications $(h=75), 11,629$ in Italy $(h=101)$, French 9,745 ( $h=102)$ and 20,143 in Germany $(h=124)$. The rate of increase in the total number of publications in Spain $(b=15)$ was lower than that observed in Italy $(b=65)$, Germany $(b=61)$ or France $(b=34)$. In the case of publications in English, the growth rate was higher for Spain $(b=37)$ than for France $(b=36)$ but lower than for Germany $(b=54)$ and Italy $(b=65)$.

Conclusions. Although the total number of publications and the observed increase are lower in Spain compared to Italy, France or Germany, the Spanish publications in Clinical Neurology shows good trend indicators with regard to publications in English and international collaboration. This improvement was associated with greater visibility as showed by the fiveyear analysis of citations received by Spanish publications.

Key words. Bibliometric analysis. Comparison between countries. Publications in neurology. 